\title{
A SUBALGEBRA CONDITION IN LIE-ADMISSIBLE ALGEBRAS
}

\author{
HYO CHUL MYUNG
}

The author wishes to present this paper in memory of his wife, Myung Mi Myung, whose untimely death occurred during the preparation of this paper. She was a trained mathematician and unselfishly encouraged the author during her illness and assisted in the preparation of the manuscript.

\begin{abstract}
Let $A$ be a finite-dimensional, flexible, Lie-admissible algebra over a field $\Phi$ of characteristic $\neq 2$. Let $S$ be a subalgebra of $A^{-}$and $H$ be a Cartan subalgebra of $S$. It is shown that $S$ is a subalgebra of $A$ if and only if $H H \subseteq S$.
\end{abstract}

For an algebra $A$, denote by $A^{-}$the algebra with multiplication $[x, y]$ $=x y-y x$ defined on the vector space $A$. If $A^{-}$is a Lie algebra then $A$ is said to be Lie-admissible. If $A$ is, in addition, a finite-dimensional flexible algebra over a field $\Phi$ then a Cartan subalgebra of $A^{-}$has played an important role for the structure of the algebra $A$ [1], [3]. Let $S$ be a subalgebra of the Lie algebra $A^{-}$. In this note, we give a condition in terms of a Cartan subalgebra of $S$ that $S$ be a subalgebra of $A$.

ThEOREM. Let $A$ be a finite-dimensional, flexible, Lie-admissible algebra over a field $\Phi$ of characteristic $\neq 2$. Let $S$ be a subalgebra of the Lie algebra $A^{-}$and $H$ be a Cartan subalgebra of $S$. Then $S$ is a subalgebra of $A$ if and only if $H H \subseteq S$.

Proof. One can assume that $\Phi$ is algebraically closed. Since $A$ is flexible and Lie-admissible, the mapping ad $x: a \rightarrow[a, x]$ is a derivation of $A$ for all $x \in A$; that is, $[a, b c]=[a, b] c+b[a, c]$ for all $a, b \in A$. Let $x, y \in A$. Then the flexible law $(x, y, y)+(y, y, x)=0$ implies $y(y x)=(x y) y-\left[x, y^{2}\right]$. Hence we get

$$
\begin{aligned}
{[[x, y], y] } & =(x y) y-(y x) y-y(x y)+y(y x)=(x y) y-2 y(x y)+y(y x) \\
& =2(x y) y-\left[x, y^{2}\right]-2 y(x y)=2[x y, y]-\left[x, y^{2}\right]=2[x, y] y-\left[x, y^{2}\right] .
\end{aligned}
$$

Therefore, we have $[x, y] y=\frac{1}{2}\left(\left[x, y^{2}\right]+[[x, y], y]\right)$.

Let $S_{\alpha}=\left\{x \in S \mid x(\operatorname{ad} h-\alpha(h) I)^{n}=0, h \in H\right.$, for some $\left.n>0\right\}$.Then since $H$ is a Cartan subalgebra of $S$, we have the root space decomposition

Received by the editors August 6, 1975 and, in revised form, December 12, 1975.

AMS (MOS) subject classifications (1970). Primary 17A20, $17 \mathrm{~B} 05$.

Key words and phrases. Lie-admissible algebra, Cartan subalgebra.

(c) American Mathematical Society 1976 
$S=\Sigma_{\alpha} S_{\alpha}$ of $S$ relative to $H$, where $H_{0}=S$ and $\left[S_{\alpha}, H\right] \subseteq S_{\alpha}$. For $\alpha \neq 0$, we first show that $S_{\alpha} H \subseteq S$. Since $\alpha \neq 0$, there exists an element $h \in H$ such that $\alpha(h) \neq 0$. Then ad $h: S_{\alpha} \rightarrow S_{\alpha}$ is surjective. For, if $[x, h]=0$ for some $x \neq 0$ in $S_{\alpha}$ then $x(\operatorname{ad} h-\alpha(h) I)^{n}=0$ implies that $\alpha(h)^{n} x=0$ and so $\alpha(h)=0$. Now, for every element $x \in S_{\alpha}$, we have

$$
[x, h] h=\frac{1}{2}\left(\left[x, h^{2}\right]+[[x, h], h]\right) \in[S, H H]+[[S, H], H] \subseteq S .
$$

Since $\left[S_{\alpha}, h\right]=S_{\alpha}$, this implies $S_{\alpha} h \subseteq S$. Let $k$ be any element in $H$. Then we have

$$
\begin{aligned}
{[x, h] k } & =[x, h k]-h[x, k]=[x, h k]-[h,[x, k]]-[x, k] h \\
& \in[S, H H]+[H,[S, H]]+\left[S_{\alpha}, H\right] h \subseteq S+S_{\alpha} h \subseteq S .
\end{aligned}
$$

Again, since $\left[S_{\alpha}, h\right]=S_{\alpha}$, this implies $S_{\alpha} H \subseteq S$ for $\alpha \neq 0$. Also, $S_{0} H=H H$ $\subseteq S$ and so we have that $S H \subseteq S$.

For any $\alpha \neq 0$, let $h$ be an element in $H$ such that $\alpha(h) \neq 0$. Let $x \in S_{\alpha}, y \in S$. Then

$$
y[x, h]=[x, y h]-[x, y] h \in[S, S H]+S H \subseteq S .
$$

Since $\left[S_{\alpha}, h\right]=S_{\alpha}$, this shows that $S S_{\alpha} \subseteq S$ for $\alpha \neq 0$. From $S S_{0}=S H$ $\subseteq S$, we have that $S S \subseteq S$, as required.

If $S$ is a subalgebra of $A^{-}$which is classical in the sense of Seligman [4], then, in view of [3, Corollary 3.4], the theorem enables us to give a condition that $S$ is a Lie algebra under the multiplication in $A$, so that a classical Lie algebra is imbedded into $A$ as a subalgebra. An element $x \in A$ is called nilpotent if $x$ is power-associative and $x^{n}=0$ for some $n>0$. We also say that a subset $M$ of $A$ is nil if every element of $M$ is nilpotent. The following is an immediate consequence of the theorem and [3, Corollary 3.4].

Corollary 1. Let $S$ be a subalgebra of $A^{-}$which is classical and $H$ be a classical Cartan subalgebra of $S$. Then $S$ is a Lie algebra under the multiplication in $A$ if and only if $H H \subseteq S$ and $H$ is nil in $A$.

In particular, if $A$ is power-associative and $A^{-}$is semisimple over $\Phi$ of characteristic 0 , it is shown that $A$ is a nilalgebra [2] and turns out to be a Lie algebra [1], [3]. The original proof of this requires that $\Phi$ is algebraically closed; however, if $\Phi$ is not algebraically closed, it can be extended to its algebraic closure. Therefore, we have

Corollary 2. Let $\Phi$ be of characteristic 0 and let $S$ be a semisimple subalgebra of $A^{-}$. Suppose that every element of $S$ is power-associative. Then $S$ is a Lie algebra under the multiplication in $A$ if and only if $S$ contains a Cartan subalgebra $H$ such that $H H \subseteq S$.

The author is indebted to the referee for many invaluable suggestions which strengthened the original version of the theorem. 


\section{REFERENCES}

1. P. J. Laufer and M. L. Tomber, Some Lie admissible algebras, Canad. J. Math. 14 (1962), 287-292. MR 25 \#104.

2. H. C. Myung, A remark on the proof of a theorem of Laufer and Tomber, Canad. J. Math. 23 (1971), 270. MR 42 \#602.

3. - Some classes of flexible Lie-admissible algebras, Trans. Amer. Math. Soc. 167 (1972), 79-88. MR 45 \#3489.

4. G. B. Seligman, Modular Lie algebras, Ergebnisse Math. Grenzgebiete, Band 40, SpringerVerlag, New York, 1967. MR 39 \#6933.

Department of Mathematics, University of Northern Iowa, Cedar Falls, Iowa 50613 